\title{
Impact of Structured Lifestyle Intervention Program with Antigravity Exercise on Glycemic Control in People with T2DM
}

\author{
T Muthu Vel. ${ }^{1}$, Karthikeyan S. $^{2 *}$ \\ DOI: https://doi.org/10.17511/ijmrr.2021.i02.04 \\ 1 T Muthu Vel, Senior Assistant Surgeon, Government Head Quarters Hospital, Tirupur, Tamil Nadu, India. \\ 2* S. Karthikeyan, Assistant Professor, Department of Orthopedics, Sivagangai Medical College, Sivagangai, Tamil Nadu, India.
}

Introduction: Diabetes mellitus (DM) is a common endocrinopathy and assumes significance for its ability to adversely affect the various internal organs. It can also derail the immune system of the affected. Abnormal insulin secretion and/or utilization, leads to hyperglycemia which has adverse effects on the heart, blood vessels, kidney, nervous system, eye and skin. To assess the effectiveness of structured vs unstructured lifestyle modification therapy in glycemic control (change in HbA1C value from baseline to the end of the intervention) in people with T2DM. Methods: This study is a randomized, controlled, parallel-group study with lifestyle modifications as intervention. Study was conducted for between Feb 2019 to Dec 2019 in the Department of General Medicine, Government Headquarters hospital, Tirupur. Results: A total of 70 participants, 30 to 60 -year-old, diagnosed with T2DM for more than a year with HbA1c levels $>6.5 \%$, on a background of oral antidiabetic agents were enrolled after obtaining the informed consent. Mean age of Group $A$ and Group B found $49.17 \pm 7.42$ and 51.92 \pm 9.1 . Male were predominant in the study. Mean weight, Mean FBS, Mean PPBS and Mean HbA1c are statistically significant between groups $(p<0.05)$ at baseline. Group B with unstructured physical activity with walking, was beneficial (HbA1c reduction $0.30 \% ; p$ $=0.024$ ), but no significant decline in HbA1c, whereas, in Group A with structured antigravity exercise, is associated with a significant HbA1c decline of $1.2 \%$. $(p=0.07865)$. Conclusion: Structured antigravity exercise training was more efficacious than unstructured physical activity in achieving controlled $\mathrm{HbA} 1 \mathrm{c}$ levels. Although both structured and unstructured training provide benefits, only the former was associated with significant reductions in HbA1c levels. Hence, T2DM patients should be advised to follow structured antigravity exercise training \& focused dietary intervention program.

Keywords: Antigravity exercise, Glycemic control, T2DM

\section{Corresponding Author}

S. Karthikeyan, Assistant Professor, Department of Orthopedics, Sivagangai Medical College, Sivagangai, Tamil Nadu, India.

Email: karthidr1976@gmail.com

\section{How to Cite this Article}

T Muthu Vel, Karthikeyan S. Impact of Structured Lifestyle Intervention Program with Antigravity Exercise on Glycemic Control in People with T2DM. Int J Med Res Rev. 2021;9(2):78-81. Available From

https://ijmrr.medresearch.in/index.php/ijmrr/article/ view/1269
To Browse

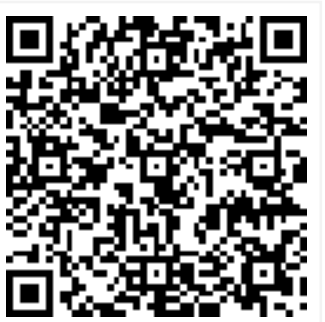

Manuscript Received 2021-02-25

Conflict of Interest No
Review Round 1 2021-03-05

Funding $\mathrm{Nil}$
Review Round 2 2021-03-10

Ethical Approval Yes
Review Round 3

Plagiarism X-checker $8 \%$
Accepted 2021-03-15

Note

(c) 2021 by T Muthu Vel, S. Karthikeyan and Published by Siddharth Health Research and Social Welfare Society. This is an Open Access article licensed under a Creative Commons Attribution 4.0 International License https://creativecommons.org/licenses/by/4.0/ unported [CC BY 4.0]. 


\section{Introduction}

Diabetes mellitus (DM) is a common endocrinopathy and assumes significance for its ability to adversely affect the various internal organs. It can also derail the immune system of the affected. Abnormal insulin secretion and/or utilization, leads to hyperglycemia which has adverse effects on the heart, blood vessels, kidney, nervous system, eye and skin.[1] The knowledge of the cutaneous signs of diabetes mellitus (DM) can be valuable to the clinicians as their observation can point towards the diagnosis of diabetes. Mostly, these cutaneous findings manifest after the diagnosis of DM, but they may appear coincidentally with its onset, or even precede diabetes by many years.[2] Diabetes mellitus (DM) is a global epidemic with an estimated worldwide prevalence of 463 million in 2019, a figure projected to reach 578 million by 2030 and 700 million by 2040 [3]. The increasing prevalence of DM is attributed to a variety of factors, including the rise in the ageing population, ethnicity, change in lifestyle, obesity, socioeconomic status, and urbanization.[4] The increasing prevalence of DM is associated with a significant increase in complications like cardiovascular diseases, endstage renal disease, neuropathy, and retinopathy. [5]National (RSSDI-ESI) and international (ADA, IDF) guidelines recommend lifestyle modifications or treatment of Type 2 Diabetes Mellitus. [6-8] although, there are guidelines that suggest lifestyle modifications, it appears that most are treated pharmacologically, and lifestyle modifications are given less importance. At presently available literature regarding the effects of lifestyle modifications, in terms of increasing the quantity and quality of physical activity on overall glycemic control independently or in combination with oral antidiabetic agents are scarce and less conclusive.

\section{Objective}

To assess the effectiveness of structured vs unstructured lifestyle modification therapy in glycemic control (change in $\mathrm{HbA1c}$ value from baseline to the end of the intervention) in people with T2DM.

\section{Materials \& Methods}

Study design: This study is a randomized, controlled, parallel-group study with lifestyle modifications as an intervention.
Study period and place: The study was conducted between Feb 2019 to Dec 2019 in the Department of General Medicine, Government Headquarters hospital, Tirupur.

Inclusion criteria: Patients diagnosed to have DM including known cases of type 2 DM already on treatment or newly detected diabetic patients.

Exclusion criteria: Patients with known comorbid conditions requiring long-term steroid therapy and patients suffering from immunocompromised conditions, type 1 DM, pulmonary tuberculosis, collagen vascular diseases, thyroid disorders, and primary dermatological conditions were excluded from the study.

Study population: A total of 70 participants, 30 to 60-year-old, diagnosed with T2DM for more than a year with $\mathrm{HbA} 1 \mathrm{c}$ levels $>6.5 \%$, on a background of oral antidiabetic agents were enrolled after obtaining the informed consent. After obtained the ethical committee have started this study. All enrolled participants underwent an educational session explaining the lifestyle modification to reduce dropout and maintain adherence. After baseline anthropometric measures of blood pressure, height, weight, BMI, blood test for lipid profile and glycated haemoglobin (HbA1c), they were allocated to respective groups using a random sampling method. The first group (Group $A \mathrm{n}=35$ ) received advice for walking for $30 \mathrm{~min} /$ day for 5 days /week along with oral antidiabetic medications. The second group (Group $\mathrm{B} n=35$ ) received a structured antigravity exercise program (15 min session) three times a day for 5 days/week, along with oral antidiabetic medications.

Statistical analysis: (Antigravity exercises: 1. Staircase climbing or 2. Nitric oxide dump - squats, alternating arm raise, non-jumping jack, or 3 . Lie down on the floor, move your hands and legs in the air). Both groups were followed up for 24 weeks.

\section{Results}

Table 1: Demographic data

\begin{tabular}{|l|l|l|l|}
\hline \multicolumn{2}{|l|}{} & \multicolumn{1}{|c|}{ Group A (n=35) } & \multicolumn{1}{c|}{ Group B (n=35) } \\
\hline \multicolumn{2}{|l|}{ Age } & $49.17 \pm 7.42$ & $51.92 \pm 9.1$ \\
\hline \multirow{3}{*}{ Sex } & Male & 21 & 19 \\
\cline { 2 - 4 } & Female & 14 & 16 \\
\hline Duration of DM & $8.17 \pm 1.21$ & $8.49 \pm 1.92$ \\
\hline Smoking & $15 / 35$ & $12 / 35$ \\
\hline Alcohol & $10 / 35$ & $08 / 35$ \\
\hline Hypertension & $27 / 35$ & $30 / 35$ \\
\hline
\end{tabular}


Totally 70 patients enrolled in our study divided into two groups. Group A walking alone along with OHA and Group B antigravity exercise along with OHA. The mean age of Group A and Group B found $49.17 \pm 7.42$ and $51.92 \pm 9.1$. Males were predominant in the study.

Table 2: Mean weight, Mean FBS, Mean PPBS and Mean HbA1c

\begin{tabular}{|c|c|c|c|c|c|}
\hline & \multicolumn{2}{|c|}{ Group A $(n=35)$} & \multicolumn{2}{|c|}{ Group B $(n=35)$} & \multirow{2}{*}{$\begin{array}{c}\mathrm{p} \\
\text { value }\end{array}$} \\
\hline & Before & After & Before & After & \\
\hline $\begin{array}{l}\text { Weight (Mean } \pm \\
\text { S.D) }\end{array}$ & $72.48 \pm 6.78$ & $80.19 \pm 7.98$ & $74.19 \pm 9.21$ & $70.41 \pm 9.78$ & $\begin{array}{l}0.0786 \\
5\end{array}$ \\
\hline $\begin{array}{l}\text { FBS (Mean } \pm \\
\text { S.D) }\end{array}$ & $\begin{array}{l}198.94 \pm 12 \\
7\end{array}$ & $174.48 \pm 9.2$ & $\begin{array}{l}214.72 \pm 21 \\
42\end{array}$ & $\begin{array}{l}177.48 \pm 8.7 \\
4\end{array}$ & 0.0047 \\
\hline $\begin{array}{l}\text { PPBS (Mean } \pm \\
\text { S.D) }\end{array}$ & $\begin{array}{l}324.12 \pm 21 . \\
42\end{array}$ & $\begin{array}{l}279.21 \pm 17 . \\
49\end{array}$ & $\begin{array}{l}359.42 \pm 23 . \\
1\end{array}$ & $\begin{array}{l}241.27 \pm 16 . \\
42\end{array}$ & $\cdot 0.0198$ \\
\hline $\begin{array}{l}\text { HbA1c (Mean } \pm \\
\text { S.D) }\end{array}$ & $11.71 \pm 2.72$ & $210.41 \pm 1.41$ & $11.24 \pm 1.79$ & $8.81 \pm 0.74$ & 0.002 \\
\hline
\end{tabular}

Mean weight, Mean FBS, Mean PPBS and Mean HbA1c are statistically significant between groups $(p<0.05)$ at baseline. Group B with unstructured physical activity with walking, was beneficial (HbA1c reduction $0.30 \% ; p=0.024$ ), but no significant decline in HbA1c, whereas, in Group A with structured antigravity exercise, is associated with a significant HbA1c decline of $1.2 \%$. $(p=0.07865)$.

\section{Discussion}

Diabetes mellitus (DM) is a common endocrinopathy and assumes significance for its ability to adversely affect the various internal organs. It can also derail the immune system of the affected. Abnormal insulin secretion and/or utilization, leads to hyperglycemia which has adverse effects on the heart, blood vessels, kidney, nervous system, eye and skin. The knowledge of the cutaneous signs of diabetes mellitus (DM) can be valuable to the clinicians as their observation can point towards the diagnosis of diabetes.

Mostly, these cutaneous findings manifest after the diagnosis of DM, but they may appear coincidentally with its onset, or even precede diabetes by many years. Anti-gravity is primarily aerobic and slightly anaerobic exercises that require the usage of significant muscle groups. In case of a reversal, the most important thing is that is you need to bring down the sugars fast. The more sugar is in control, and insulin will be in control. The more insulin will be in control, the more fats, inflammation and water everything will be balanced.
Hence doing anti-gravity is important because the number of hours sugar levels remaining above $180 \mathrm{mg} / \mathrm{dl}$, more the diabetes-related complications. Doing the staircase three times a day for 5-10 minutes each is very important in the adjustment phase.

\section{Besides sugars coming down, anti-gravity has the following benefits:}

- Increases immune function and cardiac output

- In diabetes type 1 , increases cardiorespiratory fitness, decreases insulin resistance and improves lipid levels with endothelial function

- In diabetes type 2, it reduces sugars, HbA1c, triglycerides and insulin resistance

- Improves muscle strength

- Promotes weight loss

Based on several large randomized controlled trials, current clinical guidelines $[9,10]$ acknowledge the therapeutic strength of exercise intervention. Despite this recommendation, it is estimated that only $30-40 \%$ of people with T2DM [11] engage in regular exercise program indicating that their rate of participation is significantly below. Table 1 shows the clinical characteristics of the randomized patients. Totally 70 patients enrolled in our study divided into two groups. Group A walking alone along with $\mathrm{OHA}$ and Group B antigravity exercise along with OHA. The mean age of Group $A$ and Group B found $49.17 \pm 7.42$ and 51.92 \pm 9.1 . Male predominant study. 15 Patients in Group A and 12 Patients in Group B reported actively smoke. 10 Patients in Group A and 08 Patients in Group B reported alcohol intake. 27 Patients in Group $A$ and 30 Patients in Group B reported Hypertension. The mean reported duration of diabetes mellitus in the randomized participants was $8.17 \pm 1.21$ years and $8.49 \pm 1.92$ years in Group A and Group B. Table 2 shows that Mean weight, Mean FBS, Mean PPBS and Mean $\mathrm{HbA1C}$ are statistically significant between groups $(P<0.05)$ at baseline. Group B with unstructured physical activity with walking, was beneficial (HbA1c reduction $0.30 \% ; P=0.024$ ), but no significant decline in $\mathrm{HbA1C}$, whereas, in Group $A$ with structured antigravity exercise, is associated with a significant HbA1C decline of $1.2 \%$. ( $P=$ $0.07865)$. Significant weight loss occurred in group A $2.29 \mathrm{Kgs}$ comparing to group B $2.80 \mathrm{Kgs}$. No significant difference is observed in blood pressure, total cholesterol, HDL-cholesterol and LDLcholesterol within (baseline to follow-up) and among groups. 
Many studies are showing only the walking along with OHA results but no more study conducted in antigravity exercise. This is the first study to be done. In future we will conduct the same study with more population in Type 2 DM lifestyle modification.

\section{Conclusion}

Structured antigravity exercise training was more efficacious than unstructured physical activity in achieving controlled $\mathrm{HbA} 1 \mathrm{c}$ levels. Although both structured and unstructured training provide benefits, only the former was associated with significant reductions in HbA1c levels. Hence, T2DM patients should be advised to follow structured antigravity exercise training \& a focused dietary intervention program.

\section{Contribution by authors}

$\mathrm{T}$ MuthuVel had conceptualized the study, prepared the study protocol, and conducted the data collection, analysis and manuscript writing. $T$ MuthuVel has verified all the drafts and approved the final draft; S. Karthikeyan had provided key inputs on methodology during protocol preparation, supported data compilation and analysis.

\section{Reference}

01. BK Sahay. Diabetology mellitus- Basic considerations, In- Siddharth N Shah, M Poul Anand, Aspi R Billimoria, Sanndhya A Kamat, Dilip R Karnad, YP Manjul, et al, API Textbook Of Medicine. volume 2, 8 th edition, Mumbai: the association of physicians of India. 2008;1042-3. [Crossref]

02. Amany Ali Sayed Abd El Nasser. Cutaneous Manifestations Among Diabetic Children. Egyptian Paediatric Association Gazette. 2012;2;12-5.

[Crossref]

03. IDF DIABETES ATLAS. Ninth edition 2019 [Internet]. International diabetes federation. Available from: [Article] [Crossref]

04. Thibault V, Bélanger $M$, LeBlanc $E$, Babin $L$, Halpine S, Greene B, et al. Factors that could explain the increasing prevalence of type 2 diabetes among adults in a Canadian provincea critical review and analysis. Diabetol Metab Syndr. 2016 Dec;8(1)71.

[Crossref]
05. Marín-Peñalver J], Martín-Timón I, SevillanoCollantes C, Cañizo-Gómez FJ del. Update on the treatment of type 2 diabetes mellitus. WJD. $2016 ; 7(17) 354$.

[Crossref]

06. Chawla R, Madhu SV, Makkar BM, Ghosh S, Saboo B, Kalra S. RSSDI-ESI clinical practice recommendations for the management of type 2 diabetes mellitus 2020. Indian J Endocr Metab. $2020 ; 24 ; 1-122$.

[Crossref]

07. Standards of Medical Care in Diabetes- 2020. Diabetes Care. 2020;43(Suppl. 1) care.

Available at: [Article] [Crossref]

08. International Diabetes Federation. Recommendations for Managing Type 2 Diabetes In Primary Care. 2017.

Available at: [Article] [Crossref]

09. Colberg SR, Sigal RJ, Fernhall B, Regensteiner JG, Blissmer BJ, Rubin RR, et al. American College of Sports Medicine; American Diabetes Association, Exercise and type 2 diabetes- The American College of Sports Medicine and the American Diabetes Association- Joint position statement. Diabetes Care. 2010;33;147-67.

[Crossref]

10. De Micheli A. Italian standards for diabetes mellitus 2007- Executive summary. Acta Diabetol. 2008;45;107-27.

[Crossref]

11. Plotnikoff RC, Johnson ST, Loucaides CA, Bauman AE, Karunamuni ND, Pickering MA. Population-based estimates of physical activity for adults with type 2 diabetes- A cautionary tale of potential confounding by weight status. J Obes. $2011 ; 1 ; 1-5$. [Crossref] 